\title{
Physiological underpinning of default-mode network emergence in the human infant brain
}

\author{
Qinlin $\mathrm{Yu}^{1,2}$, Minhui Ouyang ${ }^{1,2}$, John A. Detre ${ }^{2,4}$, Huiying Kang ${ }^{1,3}$, Di Hu ${ }^{1,3}$, Bo Hong ${ }^{5}$, Fang \\ Fang $^{6}$, Yun Peng ${ }^{3}$, Hao Huang ${ }^{1,2,{ }^{*}}$ \\ ${ }^{1}$ Department of Radiology, Children's Hospital of Philadelphia, Philadelphia, PA, 19104, United States; \\ ${ }^{2}$ Department of Radiology, ${ }^{4}$ Department of Neurology, Perelman School of Medicine, University of \\ Pennsylvania, Philadelphia, PA, 19104,United States; \\ ${ }^{3}$ Department of Radiology, Beijing Children's Hospital, Capital Medical University, Beijing, 100045, China; \\ ${ }^{5}$ Department of Biomedical Engineering, Tsinghua University, Beijing, 100084, China \\ ${ }^{6}$ School of Psychological and Cognitive Sciences, Peking University, Beijing, 100871,China
}

Short title: regional cerebral blood flow coupled with functional connectivity during infancy

\section{* Corresponding author}

Hao Huang, Ph.D.

Department of Radiology, Children's Hospital of Philadelphia, Perelman School of Medicine University of Pennsylvania

Philadelphia, PA, United States

Keywords: infant brain development; default mode network; perfusion; functional connectivity; metabolism; emergence 


\section{Abstract}

During infancy, rapid brain circuit maturational processes are supported by dynamic changes of regional cerebral blood flow (rCBF) to meet metabolic demands and enable functional emergence. However, the 4D spatiotemporal rCBF dynamics and mechanism of functional network emergence in infant brain are not known. Here, cutting-edge multi-modal MRI was conducted on forty-eight infants aged 0 to 24 months. With pseudo-continuous arterial-spin-labeled perfusion $\mathrm{MRI}$ of infants, heterogeneous age-related increases of rCBF were found across multiple vital functional network brain regions. Resting-state functional MRI revealed the characteristic defaultmode network (DMN) emergence in infant brain. The rCBF increase rate is significantly higher in the DMN regions than in the reference primary sensorimotor network regions. Coupled increases of rCBF and functional connectivity specifically in the DMN were found with correlation analysis and were further confirmed by data-driven permutation tests. The 4D infant rCBF maps can be used as a quantified standard reference for assessing brain physiology in typically or atypically developmental infants. These findings from multi-modal infant MRI not only offer refreshing insights into mechanism of DMN emergence driven by higher metabolic expenditure operationalized by higher local blood supply in infant brain, but also have important implications in altered network maturation in brain disorders. 


\section{Significance}

Human infancy is characterized by rapid brain circuit maturation including default-mode network (DMN) emergence. However, the physiological underpinning of DMN emergence is unknown. Here, we established a quantified standard reference of infant regional cerebral blood flow maps and found regional cerebral blood flow increases faster in the DMN than primary sensorimotor network regions during infancy. We also found strongly coupled increases of regional cerebral blood flow and network strength specifically in the DMN, suggesting emergence of the DMN is supported by faster local blood flow increase meeting extra neuronal metabolic demands for brain circuit maturation. These results shed light on fundamental mechanism of brain network emergence and have important implications in altered network maturation in brain disorders. 


\section{Introduction}

The adult human brain receives $15-20 \%$ of cardiac output despite only representing $2 \%$ of body mass $(1,2)$. In the developmental cerebral cortex, dynamic cellular and molecular processes including neurogenesis and neuronal migration $(3,4)$, synaptic formation $(5)$, dendritic arborization $(6,7)$, axonal growth $(8,9)$ and myelination $(10,11)$ are associated with a much higher fraction of cardiac output $(12,13)$ fueling the brain. Infancy is the most dynamic phase of brain development across entire lifespan. As an example, during infancy the brain size increases dramatically in parallel with rapid elaboration of new synapses, reaching $80-90 \%$ of lifetime maximum by age of year $2(14,15)$. Brain development in infancy is characterized by significant and dramatic age-related cerebral blood flow (CBF) increases after accounting for brain volume (16). Structural and functional changes of developmental brain are underlaid by rapid cellular and molecular processes precisely following a regulated spatiotemporal sequence (17-21), resulting in differential brain system maturation (22-25) following the order from primary sensorimotor to higher-order cognitive systems.

Blood perfusion of infant brain physiologically underpins functional development. Vital oxygen delivery through cerebral blood flow supports functional emergence during infancy by meeting its metabolic needs. Regional brain metabolism, including glucose utilization and oxygen consumption, is closely coupled to regional CBF (rCBF) that delivers the glucose and oxygen needed to sustain metabolic needs $(26,27)$. Infant rCBF has been conventionally measured with positron emission tomography (PET) $(28,29)$ and single-photon emission computerized tomography (SPECT) (30) methods which are not applicable to infants due to the associated exposure to radioactive tracers. By labeling the blood in internal carotid and vertebral arteries in neck and measuring downstream labeled arterial blood in brain, arterial-spin-labeled (ASL) (31, 32) perfusion $\mathrm{MRI}$ provides a method for noninvasive quantifying rCBF without requiring radioactive tracers or exogenous contrast agents. Accordingly, ASL is especially suitable for rCBF 
measurements of infants $(33,34)$ and children $(2,35)$. Phase-contrast (PC) MRI, utilizing the phase shift proportional to velocity of the blood spins, has also been used to measure global CBF of the entire brain (16). Through integration of pseudo-continuous ASL (pCASL) and PC MRI, rCBF measured from pCASL can be calibrated by global CBF from PC MRI for more accurate infant brain rCBF measurement $(33,36)$. With $\mathrm{rCBF}$ closely related to regional cerebral metabolic rate of oxygen $\left(\mathrm{CMRO}_{2}\right)$ and glucose (CMRGlu) at the resting state in human brain $(27,37-39)$, rCBF could be used as a surrogate measure of local cerebral metabolic level for resting infant brains.

The default-mode network (DMN) (26) has been widely recognized as a fundamental neurobiological system with important implication in typical and atypical developing brains (40). Unlike primary sensorimotor (SM) and visual (Vis) networks emerging relatively earlier around and before birth (41-43), emergence of the vital resting-state DMN is not well established until late infancy (44). At birth the human brain architecture already comprises a large scale of connections $(41,42,45)$ that can be measured with resting-state fMRI (rs-fMRI). Highly differentiated emergence of functional networks $(23,41-43,45)$ result from rapid neuronal growth during infancy. In general, primary sensorimotor functions emerge earlier than higher order cognitive functions during brain development. Functional networks consist of densely linked hub regions to support efficient neuronal signaling and communication. These hub regions can be delineated with data-driven independent component analysis (ICA) of rs-fMRI data and serve as functional regions-of-interests (ROIs) for testing physiology-function relationship. Meeting metabolic demands in these hub ROIs is critical for functional network maturation. In fact, spatial correlation of rCBF to the functional connectivity (FC) in these functional ROls was found in adult brains (46). Altered DMN plays a vital role in neurodevelopmental disorders such as autism (4750). Thus, understanding physiological underpinning of the DMN maturation offers invaluable insights into the mechanism of typical and atypical brain development. 
With prominent DMN emergence during infancy $(23,44)$, We hypothesized that during infancy $\mathrm{rCBF}$ increases faster in the DMN regions than in primary sensorimotor regions, where functional networks emerge earlier $(23,41-43,45,51)$. Furthermore, since rCBF is an indicator of local metabolic level of glucose and oxygen consumption of resting brains, we hypothesized faster rCBF increase in the DMN regions is strongly coupled with FC increase there to meet extra metabolic demand of DMN maturation. In this study, we acquired multi-modal MRI including both pCASL perfusion MRI and rs-fMRI of 48 infants aged 0 to 24 months to quantify rCBF and FC, respectively. T1 weighted images of all infants were also acquired for anatomical information. RCBF at the voxel level and in functional network ROls were measured to test the hypothesis of spatiotemporally differential rCBF increases during infancy. Maturation of FC in the DMN was delineated. Correlation of FC increase and rCBF increase in the DMN ROIs was tested and further confirmed with data-driven permutation analysis, the latter of which was to examine if the coupling of rCBF and FC takes place specifically in the DMN during infancy. 


\section{Results}

Faster rCBF increases in the DMN hub regions during infant brain development. The labeling plane and imaging slices of pCASL perfusion MRI of a representative infant brain, reconstructed internal carotid and vertebral arteries, and four PC MR images of the target arteries are shown in Fig. 1a. The rCBF maps of infant brains were calculated based on pCASL perfusion MRI and calibrated by PC MRI. As an overview, axial rCBF maps of typically developing brains at milestone ages of $1,6,12,18$ and 24 months are demonstrated in Fig. 1b. High quality of the rCBF maps can be appreciated by a clear contrast between white matter and gray matter. A general increase of blood flow across the brain gray matter from birth to 2 years of age is readily observed. Heterogeneous rCBF distribution at a given infant age can be appreciated from these maps. For example, higher rCBF values in primary visual cortex compared to other brain regions are clear in younger infant at around 1 month. Fig. 1b also demonstrates differential rCBF increases across brain regions. RCBF increases are prominent in the posterior cingulate cortex (PCC, a vital hub of DMN network), indicated by green arrows. On the other hand, rCBF in the visual cortex is already higher (indicated by blue arrows) than other brain regions in early infancy and increases slowly across infant development. Supplemental Information (SI) Appendix Fig. S1 quantitatively illustrates spatial inhomogeneity of rCBF distribution regardless of age, with rCBF in the Vis ROI highest, rCBF in the SM ROI lowest and rCBF in the DMN ROIs in the middle.

Fig. 2a shows cortical maps of linearly fitted rCBF values of infant brains from 0 to 24 months. Consistent with nonuniform profile of the rCBF maps observed in Fig. 1b, the threedimensionally reconstructed rCBF distribution maps in Fig. 2a are also not uniform at each milestone infant age. RCBF increases from 0 to 24 months across cortical regions are apparent, as demonstrated by relatively high rCBF-age correlation $r$ values across the cortical surface in Fig. 2b. Heterogeneity of rCBF increases across all brain voxels can be more clearly appreciated in Figs. $2 a$ and $2 b$ compared to Fig. 1b. With DMN functional network regions including PCC, medial prefrontal cortex (MPFC), inferior posterior lobule (IPL) and lateral temporal cortex (LTC) 
as well as Vis and SM network regions delineated in the SI Appendix Fig. S2b as ROIs, rCBF trajectories in Fig. 2c demonstrate that rCBF in these ROls all increase significantly with age (Vis: $r=0.53, p<10^{-4} ; \mathrm{SM}: r=0.52, p<10^{-4} ;$ DMN: $r=0.7, p<10^{-7} ;$ DMN_PCC: $r=0.66, p<10^{-6}$; DMN_MPFC: $r=0.67, p<10^{-6}$; DMN_IPL: $r=0.66, p<10^{-6} ;$ DMN_LTC: $\left.r=0.72, p<10^{-8}\right)$. Using the trajectory of primary sensorimotor (SM) (black line and circles) in Fig. 2c as a reference, rCBF increases across functional network ROls are heterogeneous (Fig. 2c). Specifically, significantly higher (all $p<0.05$, FDR corrected) rCBF increase rate was found in total DMN ROls (1.59 $\mathrm{ml} / 100 \mathrm{~g} / \mathrm{min} / \mathrm{month})$ and individual DMN ROls, including DMN_PCC (1.57 ml/100g/min/month), DMN_MPFC $(1.63 \mathrm{ml} / 100 \mathrm{~g} / \mathrm{min} / \mathrm{month})$, DMN_IPL $(1.42 \mathrm{ml} / 100 \mathrm{~g} / \mathrm{min} / \mathrm{month})$ and DMN_LTC (2.22 $\mathrm{ml} / 100 \mathrm{~g} / \mathrm{min} / \mathrm{month})$, compared to in the SM ROI $(0.85 \mathrm{ml} / 100 \mathrm{~g} / \mathrm{min} / \mathrm{month})$. Although the rCBF growth rate in the Vis $(1.27 \mathrm{ml} / 100 \mathrm{~g} / \mathrm{min} / \mathrm{month}) \mathrm{ROIs}$ is higher than that in the SM ROls, this difference was not significant $(p=0.13)$. Collectively, Figs. 1 and 2 show that the CBF increases significantly and differentially across brain regions during infancy, with rCBF in the DMN hub regions increasing faster than $\mathrm{rCBF}$ in the SM and Vis regions (Fig. 2). The 4D spatiotemporal whole-brain rCBF dynamics during infant development are presented in a movie (SI Appendix Movie S1).

Emergence of DMN during early brain development. Fig. 3a shows emergence of the DMN in typically developing brain from 0 to 24 months as measured using rs-fMRI with a PCC seed region indicated by the black dash line. At around birth (0 months), the DMN is still immature with weak FC between other DMN regions (including MPFC, IPL, and LTC) and PCC (Fig. 3a). During infant brain development from 0 to 24 months, Fig. 3a shows that the functional connectivity between MPFC, IPL or ITC and PCC gradually strengthens. Fig. 3b shows the FC-age correlation r value map. It can be appreciated from Fig. 3b that across the cortical surface relatively higher $r$ values are only located at the DMN regions (except the seed PCC). 
As shown in SI Appendix Fig. S3, only the FC within the DMN $(r=0.31, p<0.05)$, but not the Vis $(r=0.048, p=0.745)$ or SM $(r=0.087, p=0.559)$, was found to increase significantly with age, indicating significant functional development in the DMN, but not in the Vis or SM network.

Coupling between rCBF and FC within DMN during infant brain development. To test the hypothesis that $\mathrm{rCBF}$ increases in the DMN regions underlie emergence of this vital functional network, correlation between rCBF and FC was conducted across randomly selected voxels within the DMN of all infants aged 0-12 months (Fig. 4a) and all infants aged 12-24 months (Fig. $4 b)$. Significant correlations $(p<0.001)$ were found in both age groups. We further tested whether functional emergence of the DMN represented by increases of FC within the DMN (namely DMN FC) was correlated to rCBF increases specifically in the DMN regions, but not in primary sensorimotor (Vis or SM) regions. Fig. 5a shows correlations between the DMN FC and rCBF at the DMN (red lines), Vis (green lines) or SM (blue lines) voxels. The correlations between the DMN FC and averaged rCBF in the DMN, Vis or SM region are represented by thickened lines in Fig. 5a. A correlation map (Fig. 5b) between the DMN FC and rCBF across the entire brain voxels was generated. The procedures of generating this correlation map are illustrated in Fig. S4. The DMN, Vis and SM ROls in Fig. 5b were delineated with dashed red, green and blue contours, respectively, and obtained from Fig S2b. Most of significant correlations $\left(r>r_{\text {crit }}\right)$ between the DMN FC and voxel-wise rCBF were found in the voxels in the DMN regions, such as PCC, IPL and LTC, but not in the Vis or SM regions (Fig. 5b). Demonstrated in a radar plot in Fig. 5c, much higher percent of voxel with significant correlations between rCBF and the DMN FC was found in the $\operatorname{DMN}(36.7 \%, p<0.0001)$ regions, than in the SM $(14.6 \%, p>0.05)$ or $\operatorname{Vis}(5.5 \%, p>0.05)$ regions. Statistical significance of higher percent of voxels with significant correlations in the DMN $(p<0.0001)$ was confirmed using nonparametric permutation tests with 10,000 permutations. We also conducted the correlation between the Vis FC and rCBF across the brain as well as permutation test. As expected, no significant correlation between the Vis FC and rCBF can be found in any voxel in the DMN, Vis or SM ROIs, demonstrated in SI Appendix Fig. S5a. Similar 
bioRxiv preprint doi: https://doi org/10.1101/2021.02.08.430158; this version posted February 8, 2021. The copyright holder for this preprint

(which was not certified by peer review) is the author/funder, who has granted bioRxiv a license to display the preprint in perpetuity. It is made available under aCC-BY-NC-ND 4.0 International license.

analysis was also conducted for correlation between the SM FC and rCBF across the brain and percent of voxels with significant correlation was close to zero, as demonstrated in SI Appendix Fig. S5b. Combined with the results shown in Fig. 5, the results of coupling between Vis (SI Appendix Fig. S5a) or SM (Fig. S5b) FC and rCBF further demonstrated selected rCBF-FC coupling can be only found in the DMN ROls, but not in the Vis or SM network ROls. 


\section{Discussion}

We investigated physiological underpinning of functional architecture emergence of the DMN during infancy with multi-modal infant MRI. Coupling between rCBF increase and FC increase specifically in the DMN regions found in this study offers refreshing insight into the mechanism of DMN emergence during infancy. That is, DMN functional circuit emergence during infancy is supported by larger local blood flow increase in the DMN to meet metabolic demand. We also delineated the 4D brain perfusion spatiotemporal dynamics characterized with heterogeneous rCBF distribution across brain regions at a specific age and differential agedependent rCBF increases across brain regions during infant development. Elucidating the ontogeny of infant brain physiology (i.e. spatiotemporal dynamics of the infant rCBF) could greatly advance current understanding of general principles of normal development. The 4D rCBF profiles can also be used as a quantified standard reference for detecting rCBF alterations (e.g. the z scores) of atypically developing brains.

Recent rs-fMRI studies have identified gradient on emergence of functional networks during early development. Differential emergence of these functional networks is characterized by different onset time as well as different maturational rate of various brain functions in a given developmental period. Primary sensory and motor functional networks, such as the SM and Vis networks, appear earlier before or around birth $(41-43,45,51)$. Other functional networks involved in heteromodal functions appear later. The $\operatorname{DMN}(26,52-56)$ is a higher-order functional network. Cao et al found rapid maturation of primary sensorimotor functional systems in last several weeks before normal time of birth while the DMN remained immature during that period (41). Smyser et al (43) also found that SM and Vis functional networks mature earlier and demonstrate adult-like pattern for preterm neonate brain, with the DMN much immature and incomplete around birth. These previous studies suggest that significant functional maturation in primary sensorimotor networks occur earlier in preterm and perinatal developmental period (41-43) compared to 0-24month infancy focused in the present study. Distinguished functional network developmental 
pattern characterized by the DMN emergence is demonstrated in human infancy. Consistent to the literature (44), functional network emergence in the DMN from 0 to 24 months was found in Fig. 3 of this study. Furthermore, SI Appendix Fig S3 demonstrated significant increase of FC only in the DMN, but not in primary sensorimotor system that already developed before normal time of birth. The differentiated development of functional connectivity from rs-fMRI suggested in the literature (41-45) is strikingly consistent to differentiated rCBF increases (Fig. 1 and Fig. 2) found in the present study.

Energy consumed in the brain is primarily yielded by aerobic glycolysis and oxidative phosphorylation $(26,27)$, where glucose and oxygen are two primary molecules for energy metabolism. Glucose consumed by infant brain represents $30 \%$ total amount of glucose $(12,13)$, more than $15-20 \%$ typically seen in adult brain $(1,2)$. The cerebral metabolic rate for glucose (CMRGlu) and oxygen (CMRO2) are direct measures of the rate of energy consumption, which parallel the proliferation of synapses in brain during infancy (12). RCBF delivering glucose and oxygen for energy metabolism in the brain is closely related to CMRGlu and CMRO2 and can serve as a surrogate of these two measurements $(27,37-39)$. In the PET study (28) using CMRGlu measurements, it was found that the local CMRGlu in the sensorimotor cortex almost reaches the highest level in early infancy and then plateaus during rest of infancy, consistent with relatively small changes of rCBF in later infancy in primary sensorimotor ROls found in this study (Fig. 2). Measured with PC MRI (16), the global CBF increases dramatically during infancy with global CBF at 18 months almost 5 times of the CBF around birth. Consistent with significant global CBF increase and differential brain functional maturation in infancy, rCBF increase pattern (Fig. 2) follows functional network maturation pattern (Fig. 3) to meet extra metabolic needs of specific functional network emergence.

Heterogeneous rCBF distribution across the brain regions (Fig. 1) at a specific age and differential maturational rates of the rCBF (Fig. 2) from 0 to 24 months are consistent with spatially 
distinctive structural (e.g. review in (57)) and functional (e.g. review in (23)) maturational processes. In developmental brains, cellular processes supporting differential functional emergence require extra oxygen and glucose delivery through cerebral blood flow to meet the metabolic demand. In Hebb's principle, "neurons that fire together wire together". Through the synaptogenesis in neuronal maturation, the neurons within a certain functional network system tend to have more synchronized activity in a more mature stage than in an immature stage. As shown in the diagram in Fig. 6, cellular activities in developmental brains, such as synaptogenesis critical for brain circuit formation, need extra energy more than that in the stable and matured stage. Neurons do not have internal reserves of energy in the form of sugar or oxygen. The demand of extra energy requires an increase in rCBF to deliver more oxygen and glucose for formation of brain networks. In the context of infant brain development, there is a cascade of events of $\mathrm{CBF}$ increase, CMRO2 and CMRGlu increase, synaptogenesis increase and synaptic efficacy increase, blood oxygenation level dependent (BOLD) signal synchronization increase, and functional connectivity increase, shown in the bottom of Fig. 6. Spatial correlation of rCBF to the FC in the functional network ROls was found in adults (46). Higher rCBF has also been found in the DMN in children 6 to 20 years of age (58). Consistent with the diagram shown in Fig. 6, Fig. 4 revealed significant correlation between $\mathrm{FC}$ and $\mathrm{rCBF}$ in the DMN network, and Fig. 5 revealed this significant correlation between FC and rCBF is specific in the DMN network, but not in the primary Vis or SM networks. These results (Fig. 4 and Fig. 5) confirmed the hypothesis that faster rCBF increase in the DMN underlies emergence of the DMN reflected by significant FC increases. Such physiology-function relationship may shed light on fundamental physiological underpinnings of DMN development.

Several technical considerations should be noted. First, present study benefits from multimodal MRI allowing measuring functional network emergence and rCBF of the same cohort of infants. Simultaneous rCBF and FC measurements enabled us to probe relationship of brain physiology and function during infant development. Second, a nonparametric permutation 
analysis without a prior hypothesis at the voxel level across the whole brain was conducted to confirm the coupling of rCBF and FC is specific in the DMN regions (Fig. 5), not in the primary sensorimotor (Vis or SM) regions (Fig. S5), enhancing the robustness of the results on physiological underpinning of functional network emergence in the DMN. Third, the pCASL perfusion MRI was calibrated by PC MRI so that the errors caused by varying labeling efficiency among infant subjects can be ameliorated. This calibration process therefore enhanced accuracy of rCBF measurements for their potential use as a standard reference. Fourth, the ROIs for rCBF measurements were obtained by data-driven ICA analysis of the same sub-cohort of infant subjects aged 12 to 24 months instead of transferred ROls from certain brain parcellation atlases. Since most of parcellation atlases were built based on adult brain data and all these atlases were established based on other subject groups, ROls delineated from the same cohort improve accuracy of coupling analysis. There are several limitations in the present study that can be improved in future investigations. All data were acquired from a cross-sectional cohort. To minimize the inter-subject variability, future study with a longitudinal cohort of infants is warranted. With relatively small size of infant brains, spatial resolution of the $\mathrm{pCASL}$ and $\mathrm{rs}-\mathrm{fMRI}$ can be further improved too to improve imaging measurement accuracy. To further improve the statistical power, larger infant sample size will be beneficial in the future studies. Finally, although the rCBF from pCASL perfusion MRI is highly correlated with the CMRO2 and CMRGlu measured from PET, rCBF is not direct measurement of rate of energy consumption. Physiology-function relationship studies in infants could benefit from development of novel noninvasive MR imaging methods for measuring CMRO2 or CMRGlu as an alternative to PET which uses radioactive tracer.

\section{Conclusion}

Based on rCBF and FC measurements from multimodal MRI in developmental brains of same cohort of infants, our results inform the mechanism of DMN emergence during infancy from physiological aspect. The age-specific whole-brain rCBF maps and rCBF maturational charts in 
bioRxiv preprint doi: https://doi.org/10.1101/2021.02.08.430158; this version posted February 8, 2021. The copyright holder for this preprint

(which was not certified by peer review) is the author/funder, who has granted bioRxiv a license to display the preprint in perpetuity. It is made available under aCC-BY-NC-ND 4.0 International license.

all brain regions serve as a standardized reference of infant brain physiology for precision medicine. The rCBF-FC coupling results shed light on fundamental physiology-function relationship during functional network maturation and have important implications in altered network maturation in developmental brain disorders. 


\section{Materials and Methods}

Infant Subjects. Forty-eight infants (30 males) aged 0-24 months (14.6 \pm 6.32 months) were recruited at Beijing Children's Hospital. These infants were referred to MR imaging due to seizures with fever $(n=21)$, convulsion $(n=17)$, diarrhea $(n=9)$, or sexual precocity $(n=1)$. All infants had normal neurological examinations documented in medical record. The exclusion criteria include known nervous system disease, or history of neurodevelopmental or systemic illness. Every infant's parents provided signed consent and the protocol was approved by Beijing Children's Hospital Research Ethics Committee.

Data acquisition. All infant MR scans including pCASL, PC MRI, rs-fMRI and structural MRI were acquired with the same 3T Philips Achieva system under sedation. See SI methods for further details.

Measurement of rCBF with pCASL perfusion MRI and calibrated by PC MRI. After head motion correction of the pCASL perfusion MRI, we estimated rCBF using the protocol similar to that in our previous publication (33). Briefly, rCBF was measured using a model described in ASL white paper (31):

$$
\mathrm{rCBF}=\frac{6000 \cdot \lambda \cdot \Delta M \cdot e^{\frac{P L D}{T_{1 a}}}}{2 \cdot \alpha \cdot M_{b}^{0} \cdot T_{1 a} \cdot\left(1-e^{\frac{- \text { LabelDur }}{T_{1 a}}}\right)}[\mathrm{ml} / 100 \mathrm{~g} / \mathrm{min}]
$$

where $\Delta \mathrm{M}$ is the dynamic-averaged signal intensity difference between in the control and label images; $\lambda$, the blood-brain partition coefficient, is $0.9 \mathrm{ml} / \mathrm{g}(59)$; PLD, the post labeling delay time, is the cumulation of $1650 \mathrm{~ms}$ and the delayed time between slices; LabelDur, the labeling duration, is $1600 \mathrm{~ms}$; $\alpha$, the labeling efficiency, is 0.86 predicted by the fitting between labeling efficiency and blood velocity in the previous study (36); $\mathrm{T}_{1 \mathrm{a}}, \mathrm{T}_{1}$ of arterial blood, is $1800 \mathrm{~ms}(60,61)$. The value of equilibrium magnetization of brain tissue $\left(M_{b}^{0}\right)$ was obtained from an auxiliary scan with identical readout module of pCASL except labeling. The labeling efficiency $\alpha$ can vary considerably across participants, especially in infants. Thus, we used PC MRI to estimate and 
calibrate rCBF measures, as described previously $(33,36)$. To calibrate rCBF, global CBF from PC MRI was calculated as follows:

$$
f_{P C, A V G}=\int v d A /(\rho * \text { brain volume })
$$

where $v$ is the blood flow velocity in the ICAs and VAs; $A$ is the cross-sectional area of the blood vessel with the unit $\mathrm{mm}^{2}$; and the brain tissue density $\rho$ is assumed as $1.06 \mathrm{~g} / \mathrm{mL}(59,62)$. Brain volume was measured from the T1-weighted image as parenchyma volume (gray matter + white matter volume). RCBF was calibrated by applying the scalar factor making averaged rCBF equal to global CBF from PC MRI.

Multi-modal image registration to a customized template space from all subjects. To integrate perfusion MRI and rs-fMRI data of all subjects, images of different acquisition modality from all subjects across different ages were registered to a customized template space with details in SI methods.

Characterization of age-dependent changes of rCBF and FC. After multi-modal images from all subjects were registered in the same template space, age-dependent rCBF and FC changes were characterized with a linear model: $y(t)=\alpha+\beta t+\varepsilon$, where y was rCBF or FC measurement at a certain ROI or voxel, $\alpha$ and $\beta$ were intercepts and slopes for $y, t$ was the infant age in months and $\varepsilon$ was the error term. See SI methods for further details.

Coupling between rCBF and FC during the infant brain development. Coupling between rCBF and FC in the DMN was conducted with voxel-wise approach. The FC of a voxel in the DMN was the average of correlations of rs-fMRI BOLD signal between this voxel and all other DMN voxels. All infants were divided into two groups based on their ages, 0-12 months and 12-24 months. 4000 voxels were randomly chosen from the DMN voxels of all subjects in each age group for regression analysis. Since the variance of both FC and rCBF cannot be ignored in this study, Deming regression (63) was used to fit the trendline of coupling between FC and rCBF. We further tested whether significant FC-rCBF coupling was specifically localized in the DMN, but not in primary sensorimotor (Vis or SM) regions. FC within a specific network was calculated by 
averaged FC of all voxels in this network. As demonstrated in Fig. S4, the correlation between FC within a network and the $\mathrm{rCBF}$ at each voxel resulted in a whole-brain $r$ map (e.g. Fig 5b). A nonparametric permutation test was then applied to evaluate the significance of rCBF-FC correlation in the ROls of a specific brain network (e.g. DMN, SM or Vis). The null hypothesis is that the voxels with significant correlation $(r>0.28)$ between $\mathrm{rCBF}$ and FC are distributed evenly in the brain. To test the null hypothesis, we resampled the correlation coefficient $r$ of all brain voxels randomly for 10,000 times to build 10,000 whole-brain correlation coefficient distribution maps. The FC-rCBF correlation was considered significant in certain brain network ROls if the number of observed significant voxels in the network ROls is higher than the number of significant voxels corresponding to 95th percentile in the permutation tests.

Acknowledgements. This work was supported by grants from National Institute of Health to Dr Huang (MH092535, MH092535-S1 and HD086984), Chinese Ministry of Science and Technology to Dr Fang (MOST 2015CB351800) and National Natural Science Foundation of China (NSFC) to Dr Fang (31421003 and 31671168) and Dr Peng (81671651). 


\section{References}

1. G. J. Bouma, J. P. Muizelaar, Relationship between cardiac output and cerebral blood flow in patients with intact and with impaired autoregulation. $J$ Neurosurg $73,368-374$ (1990).

2. T. D. Satterthwaite et al., Impact of puberty on the evolution of cerebral perfusion during adolescence. Proc. Natt. Acad. Sci. U.S.A. 111, 8643-8648 (2014).

3. R. L. Sidman, P. Rakic, Neuronal migration, with special reference to developing human brain: a review. Brain Res. 62, 1-35 (1973).

4. P. Rakic, Radial versus tangential migration of neuronal clones in the developing cerebral cortex. Proc. Natl. Acad. Sci. U.S.A. 92, 11323 (1995).

5. P. R. Huttenlocher, A. S. Dabholkar, Regional differences in synaptogenesis in human cerebral cortex. J. Comp. Neurol. 387, 167-178 (1997).

6. I. Bystron, C. Blakemore, P. Rakic, Development of the human cerebral cortex: Boulder Committee revisited. Nat. Rev. Neurosci. 9, 110-122 (2008).

7. M. Ouyang et al., Differential cortical microstructural maturation in the preterm human brain with diffusion kurtosis and tensor imaging. Proc. Natl. Acad. Sci. U.S.A. 116, 46814688 (2019).

8. R. L. Haynes et al., Axonal development in the cerebral white matter of the human fetus and infant. J. Comp. Neurol. 484, 156-167 (2005).

9. G. M. Innocenti, D. J. Price, Exuberance in the development of cortical networks. Nat. Rev. Neurosci. 6, 955-965 (2005).

10. D. J. Miller et al., Prolonged myelination in human neocortical evolution. Proc. Natl. Acad. Sci. U.S.A. 109, 16480-16485 (2012).

11. P. Yakovlev, The myelogenetic cycles of regional maturation of the brain. Regional development of the brain in early life, 3-70 (1967).

12. M. E. Raichle, Two views of brain function. Trends Cogn. Sci. 14, 180-190 (2010).

13. G. Settergren et al., Cerebral blood flow and exchange of oxygen, glucose, ketone bodies, lactate, pyruvate and mino acides in infants. Acta Pediatrica Scand. 65, 343-353 (1976).

14. R. C. Knickmeyer et al., A structural MRI study of human brain development from birth to 2 years. J. Neurosci. 28, 12176-12182 (2008).

15. A. Pfefferbaum et al., A quantitative magnetic resonance imaging study of changes in brain morphology from infancy to late adulthood. J. Neurol. 51, 874-887 (1994).

16. P. Liu et al., Assessment of cerebral blood flow in neonates and infants: a phase-contrast MRI study. Neurolmage 185, 926-933 (2019).

17. P. R. Huttenlocher, A. S. Dabholkar, Regional differences in synaptogenesis in human cerebral cortex. J Comp Neurol 387, 167-178.

18. RL. Sidman, P Rakic, Development of the human central nervous system. Histology and histopathology of the nervous system, eds Haymaker W, Adams RD(Charles C. Thomas, Springfield, IL), pp 3-I45 (1982).

19. J. C. Silbereis, S. Pochareddy, Y. Zhu, M. Li, N. Sestan, The cellular and molecular landscapes of the developing human central nervous system. Neuron 89, 248-268 (2016).

20. H. Huang et al., Coupling diffusion imaging with histological and gene expression analysis to examine the dynamics of cortical areas across the fetal period of human brain development. Cereb Cortex 23, 2620-2631 (2013).

21. S. L. Andersen, Trajectories of brain development: point of vulnerability or window of opportunity? Neurosci Biobehav Rev. 27, 3-18 (2003).

22. G. Z. Tau, B. S. Peterson, Normal development of brain circuits. Neuropsychopharmacology 35, 147 (2010).

23. M. Cao, H. Huang, Y. He, Developmental connectomics from infancy through early childhood. Trends Neurosci. 40, 494-506 (2017). 
24. H. Huang, L. Vasung, Gaining insight of fetal brain development with diffusion MRI and histology. Int. J. Dev. Neurosci. 32, 11-22 (2014).

25. Q. Yu et al., Structural development of human fetal and preterm brain crotical plate based on population-averaged templates. Cereb Cortex 26, 4381-4391 (2016).

26. M. E. Raichle et al., A default mode of brain function. Proc. Natl. Acad. Sci. U.S.A. 98, 676-682 (2001).

27. S. N. Vaishnavi et al., Regional aerobic glycolysis in the human brain. Proc. Natl. Acad. Sci. U.S.A. 107, 17757-17762 (2010).

28. H. T. Chugani, M. E. Phelps, Maturational changes in cerebral function in infants determined by 18FDG positron emission tomography. Science 231, 840-843 (1986).

29. H. T. Chugani, M. E. Phelps, J. C. Mazziotta, Positron emission tomography study of human brain functional development. Ann. Neurol. 22, 487-497 (1987).

30. C. Chiron et al., Changes in regional cerebral blood flow during brain maturation in children and adolescents. J. Nucl. Med.: official publication, Society of Nuclear Medicine 33, 696703 (1992).

31. D. C. Alsop et al., Recommended implementation of arterial spin-labeled perfusion MRI for clinical applications: A consensus of the ISMRM perfusion study group and the European consortium for ASL in dementia. Magn Reson Med. 73, 102-116 (2015).

32. J. A. Detre, D. C. Alsop, Perfusion magnetic resonance imaging with continuous arterial spin labeling: methods and clinical applications in the central nervous system. Eur J Radiol. 30, 115-124 (1999).

33. M. Ouyang et al., Heterogeneous increases of regional cerebral blood flow during preterm brain development: Preliminary assessment with pseudo-continuous arterial spin labeled perfusion MRI. Neurolmage 147, 233-242 (2017).

34. Z. Wang et al., Assessment of functional development in normal infant brain using arterial spin labeled perfusion MRI. Neuroimage 39, 973-978 (2008).

35. V. Jain et al., Longitudinal reproducibility and accuracy of pseudo-continuous arterial spinlabeled perfusion MR imaging in typically developing children. Radiology 263, 527-536 (2012).

36. S. Aslan et al., Estimation of labeling efficiency in pseudocontinuous arterial spin labeling. Magn Reson Med. 63, 765-771 (2010).

37. P. T. Fox, M. E. Raichle, Focal physiological uncoupling of cerebral blood flow and oxidative metabolism during somatosensory stimulation in human subjects. Proc. Natl. Acad. Sci. U.S.A. 83, 1140-1144 (1986).

38. R. C. Gur et al., Regional differences in the coupling between resting cerebral blood flow and metabolism may indicate action preparedness as a default state. Cereb. Cortex 19, 375-382 (2009).

39. O. B. Paulson, S. G. Hasselbalch, E. Rostrup, G. M. Knudsen, D. Pelligrino, Cerebral blood flow response to functional activation. J. Cereb. Blood Flow Metab. 30, 2-14 (2010).

40. R. L. Buckner, J. R. Andrews-Hanna, D. L. Schacter, The brain's default network: anatomy, function, and relevance to disease. Ann. N. Y. Acad. Sci. 1124, 1-38 (2008).

41. M. Cao et al., Early development of functional network segregation revealed by connectomic analysis of the preterm human brain. Cereb. Cortex 27, 1949-1963 (2017).

42. V. Doria et al., Emergence of resting state networks in the preterm human brain. Proc. Natl. Acad. Sci. U.S.A. 107, 20015-20020 (2010).

43. C. D. Smyser et al., Longitudinal analysis of neural network development in preterm infants. Cereb. Cortex 20, 2852-2862 (2010).

44. W. Gao et al., Evidence on the emergence of the brain's default network from 2-week-old to 2-year-old healthy pediatric subjects. Proc. Natl. Acad. Sci. U.S.A. 106, 6790-6795 (2009). 
45. P. Fransson et al., Resting-state networks in the infant brain. Proc. Natl. Acad. Sci. U.S.A. 104, 15531-15536 (2007).

46. X. Liang, Q. Zou, Y. He, Y. Yang, Coupling of functional connectivity and regional cerebral blood flow reveals a physiological basis for network hubs of the human brain. Proc. Natl. Acad. Sci. U.S.A. 110, 1929-1934 (2013).

47. K. A. Doyle-Thomas et al., Atypical functional brain connectivity during rest in autism spectrum disorders. Ann. Neurol. 77, 866-876 (2015).

48. C. J. Lynch et al., Default mode network in childhood autism: posteromedial cortex heterogeneity and relationship with social deficits. Biol. Psychiatry 74, 212-219 (2013).

49. A. Padmanabhan, C. J. Lynch, M. Schaer, V. Menon, The default mode network in autism. Biol Psychiatry Cogn Neurosci Neuroimaging 2, 476-486 (2017).

50. S. D. Washington et al., Dysmaturation of the default mode network in autism. Hum Brain Mapp 35, 1284-1296 (2014).

51. Q. Peng et al., Regularized-Ncut: Robust and homogeneous functional parcellation of neonate and adult brain networks. Artif Intell Med 106, 101872 (2020).

52. M. D. Fox, A. Z. Snyder, J. L. Vincent, M. E. Raichle, Intrinsic fluctuations within cortical systems account for intertrial variability in human behavior. Neuron 56, 171-184 (2007).

53. M. D. Greicius, B. Krasnow, A. L. Reiss, V. Menon, Functional connectivity in the resting brain: a network analysis of the default mode hypothesis. Proc. Natl. Acad. Sci. U.S.A. 100, 253-258 (2003).

54. M. D. Greicius, K. Supekar, V. Menon, R. F. Dougherty, Resting-state functional connectivity reflects structural connectivity in the default mode network. Cereb. Cortex 19, $72-78$ (2009).

55. M. E. Raichle, The brain's default mode network. Annu. Rev. Neurosci. 38, 433-447 (2015).

56. S. M. Smith et al., Correspondence of the brain's functional architecture during activation and rest. Proc. Natl. Acad. Sci. U.S.A. 106, 13040-13045 (2009).

57. M. Ouyang, J. Dubois, Q. Yu, P. Mukherjee, H. Huang, Delineation of early brain development from fetuses to infants with diffusion MRI and beyond. Neuroimage 185, 836850 (2019).

58. F. Liu et al., Resting state cerebral blood flow with arterial spin labeling MRI in developing human brains. Eur. J. Paediatr. Neurol. 22, 642-651 (2018).

59. P. Herscovitch, M. E. Raichle, What is the correct value for the brain-blood partition coefficient for water? J. Cereb. Blood Flow Metab. 5, 65-69 (1985).

60. P. Liu et al., T1 and T2 values of human neonatal blood at 3 Tesla: dependence on hematocrit, oxygenation, and temperature. Magn Reson Med. 75, 1730-1735 (2016).

61. M. Varela et al., A method for rapid in vivo measurement of blood T1. NMR Biomed. 24, 80-88 (2011).

62. J. Dittmer, R. Dawson, The isolation of a new lipid, triphosphoinositide, and monophosphoinositide from ox brain. Biochemical Journal 81, 535 (1961).

63. W.E. Deming, Statistical adjutement of data. Wiley, NY (1943). 

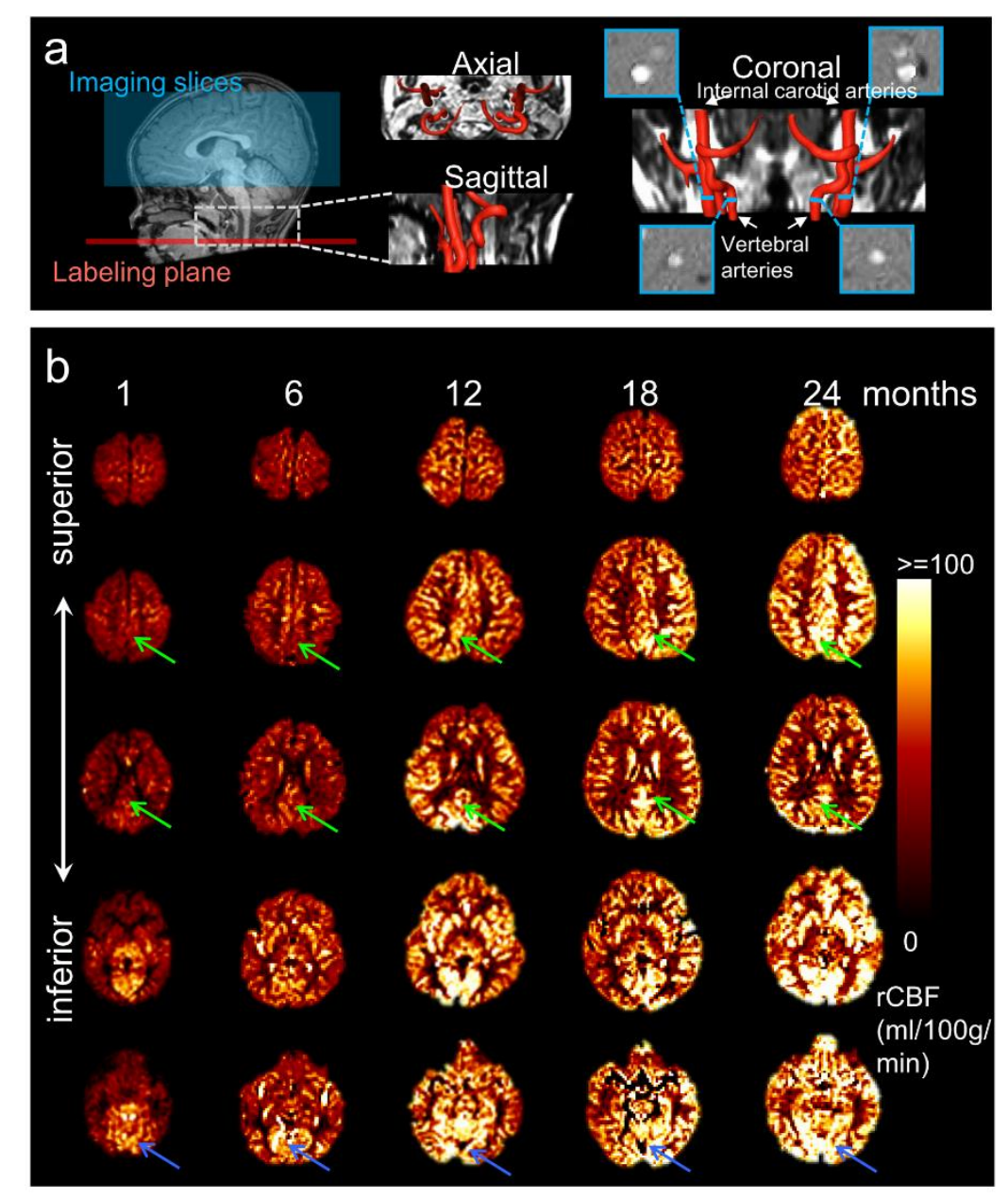

Fig. 1. (a) Labeling plane (red line) and imaging volume (blue box) of pseudo-continuous arterialspin-labeled perfusion MR imaging are shown on the mid-sagittal slice of T1-weighted image of a representative infant on the left panels. Axial and sagittal view of MR angiography with reconstructed internal carotid and vertebral arteries are shown in the middle of the panel a. On the right of the panel a, the coronal view of the reconstructed arteries is placed in the middle with four slices (shown as blue bars) of the phase-contrast (PC) MR scans positioned perpendicular to the respective feeding arteries. The PC MR images are shown on the four panels surrounding the coronal view of the angiography. These PC MR images measure the global cerebral blood flow of internal carotid and vertebral arteries and are used to calibrate regional cerebral blood flow (rCBF). (b) rCBF maps of representative typically developing (TD) infant brains at 1, 6, 12, 18 and 24 months from left to right. Axial slices of rCBF maps from inferior to superior are shown from 
bioRxiv preprint doi: https://doi.org/10.1101/2021.02.08.430158; this version posted February 8, 2021. The copyright holder for this preprint

(which was not certified by peer review) is the author/funder, who has granted bioRxiv a license to display the preprint in perpetuity. It is made available under aCC-BY-NC-ND 4.0 International license.

bottom to top of the panel $b$ for each TD infant brain. Green arrows point to the posterior cingulate cortex (a hub of the DMN network) characterized by relatively lower rCBF at early infancy and prominent rCBF increases from 1 to 24 months. Blue arrows point to the visual cortex characterized by relatively higher rCBF at early infancy and relatively mild rCBF increase from 1 to 24 months. 

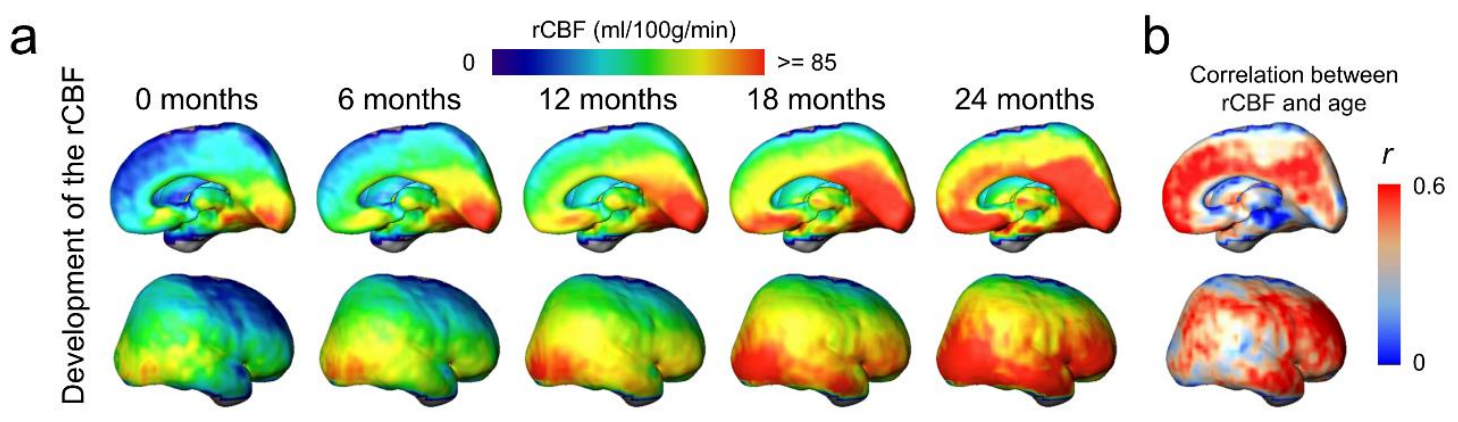

C

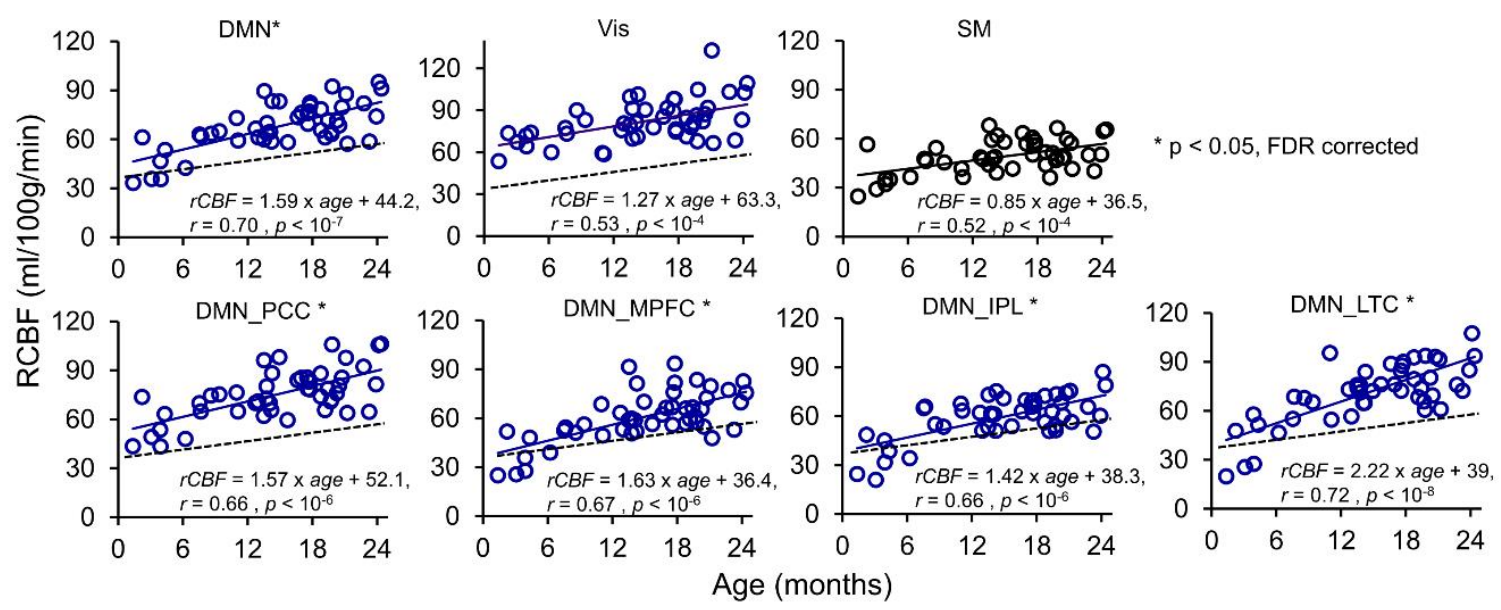

Fig. 2. (a) Medial (top row) and lateral (bottom row) views of fitted rCBF profiles of the infant brain at $0,6,12,18$ and 24 months in the custom-made infant template space demonstrate heterogeneous rCBF increase across the brain regions. (b) Medial (top) and lateral (bottom) views of rCBF-age correlation coefficient ( $r$ ) map are demonstrated. (c) The scatterplots of rCBF measurements in the primary sensorimotor (SM) network (black circle and black line), visual (Vis) network (blue circle and blue line), and total and individual default-mode network (DMN) hub regions (DMN_MPFC, DMN_PCC, DMN_IPL and DMN_LTC) (blue circle and blue line) demonstrate differential rCBF increase rates. ${ }^{*}$ next to network name in each plot indicates significant $(F D R$-corrected $p<0.05$ ) differences of $\mathrm{rCBF}$ trajectory slope from that of $\mathrm{SM}$ used as a reference and shown in a black dashed line. Abbreviations of DMN subregions: IPL: inferior posterior lobule; LTC: lateral temporal cortex; MPFC: medial prefrontal cortex; PCC: posterior cingulate cortex. 


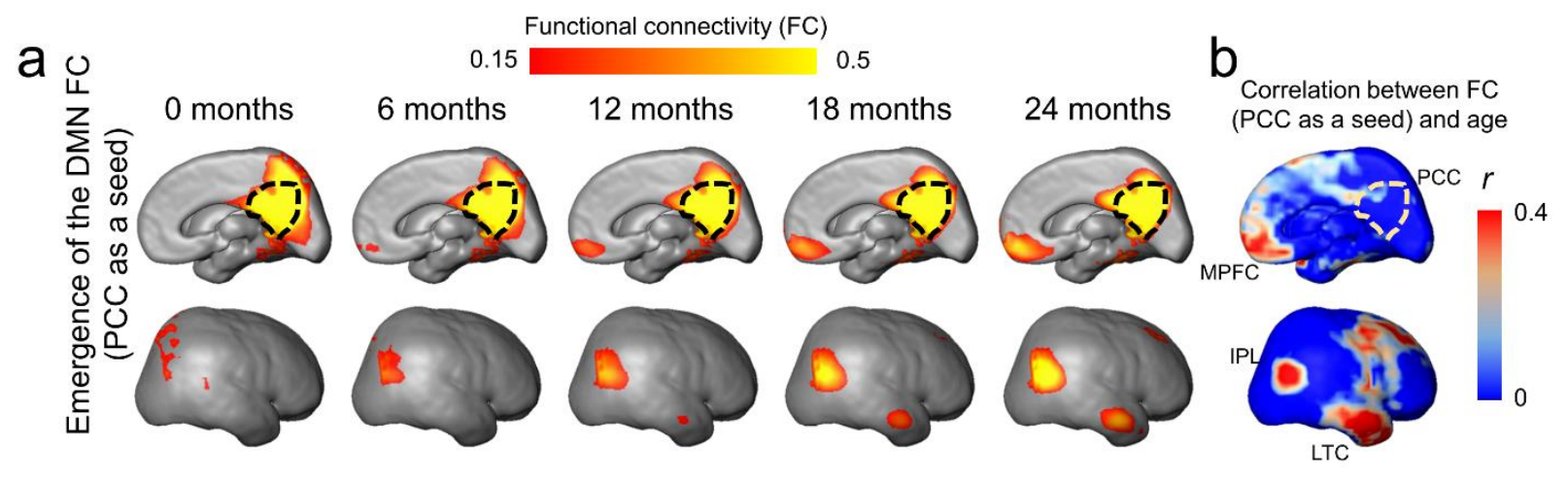

Fig. 3. Emergence of functional connectivity (FC) within the default-mode network (DMN) during infancy is demonstrated by the maps of the DMN FC (posterior cingulate cortex (PCC) as a seed) at representative ages from 0 to 24 months (a) and the map of correlation coefficient of FC (PCC as a seed) and age (b). In (a), gradually emerging FC of other DMN regions (including MPFC, IPL and LTC) to the PCC from 0 to 24 months can be appreciated. The PCC is delineated by the black dashed contour. In (b), stronger correlation between FC (PCC as a seed) and age is localized in DMN subregions IPL, ITL and MPFC. See legend of Figure 2 for abbreviations of the DMN subregions. 

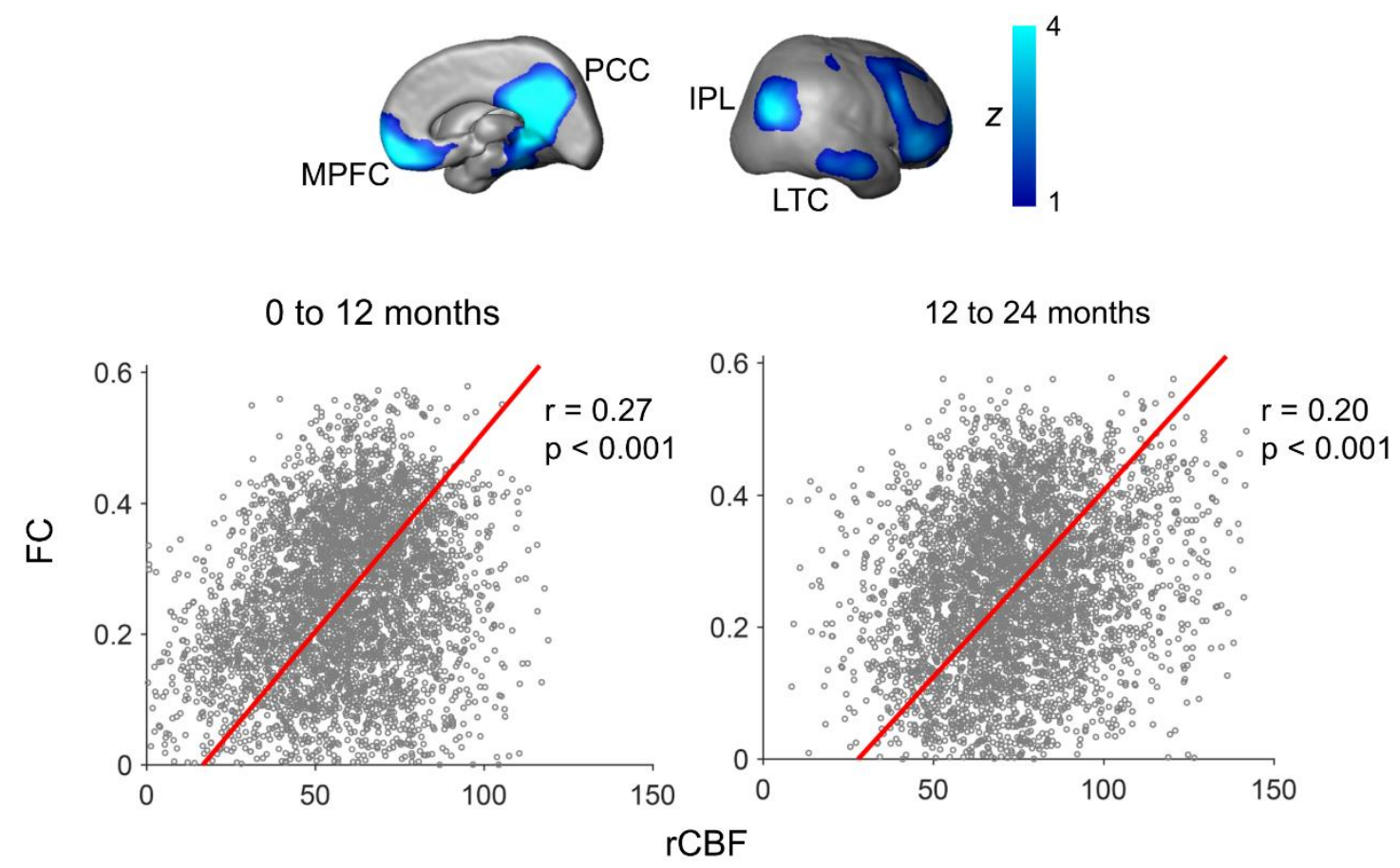

Fig. 4. Significant correlation of regional cerebral blood flow ( $\mathrm{rCBF}$ ) and functional connectivity (FC) at randomly selected 4000 voxels within the default-mode network (DMN) for both infants aged $0-12$ months $(p<0.001$, left scatter plot) and infants aged 12-24 months $(p<0.001$, right scatter plot). FC is the average of FC of a certain DMN voxel to all other DMN voxels. The DMN regions of interests obtained from a data-driven independent component analysis of resting-state fMRI of the 12-24-month infant cohort are shown on the top panels as an anatomical reference. See legend of Figure 2 for abbreviations of the DMN subregions. 
a

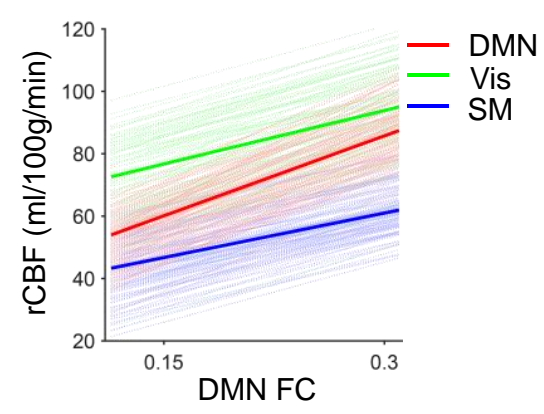

b Correlation between the DMN $\mathrm{FC}$ and $\mathrm{rCBF}$ across the brain

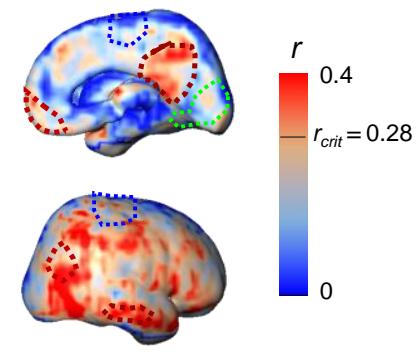

C

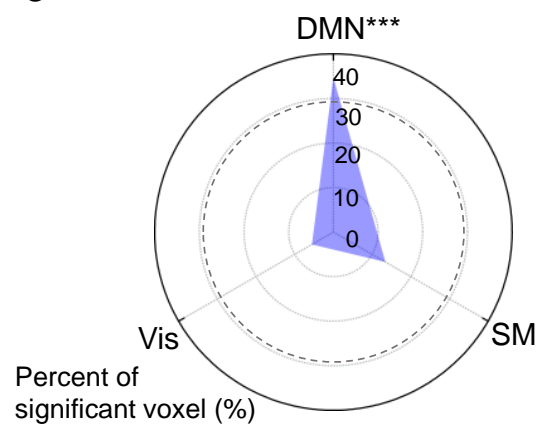

Fig. 5. (a) Correlation of intra-default-mode-network functional connectivity (DMN FC) and regional cerebral blood flow (rCBF) at randomly selected voxels in the default-mode (DMN, light red lines), visual (Vis, light green lines) and sensorimotor (SM, light blue lines) network regions. Correlations of DMN FC and averaged rCBF in the DMN, Vis and SM network regions are shown as thickened red, green and blue lines, respectively. (b) Coupling between the DMN FC and rCBF across the brain can be appreciated by distribution of voxel-wise correlation coefficient $(r)$ obtained from correlation between DMN FC and rCBF at each voxel. The short black line in the color bar indicates critical $r$ value $r_{\text {crit }}$ corresponding to $p=0.05$. Higher $r$ values can be appreciated in the DMN hub regions including posterior cingulate cortex (PCC), medial prefrontal cortex (MPFC), inferior posterior lobule (IPL) and lateral temporal cortex (LTC) with their boundaries delineated by the dashed dark red contours (from Fig. S2b). Dashed green and blue contours (also from Fig. S2b) delineate the Vis and SM network regions, respectively. (c) Radar plot shows significant correlation between rCBF and intra-DMN FC in the DMN network $(36.7 \%, p<0.0001)$, but not in the SM $(14.6 \%, p>0.05)$ or Vis $(5.5 \%, p>0.05)$ networks. The radius represents the percent of the voxels with significant correlations between intra-DMN FC and rCBF in DMN, Vis and SM network regions, respectively. The dashed line circle indicates critical percent of significant voxels with $\mathrm{p}=0.05$ from 10,000 permutation tests. ${ }^{* * *}$ indicates $\mathrm{p}<0.0001$. 


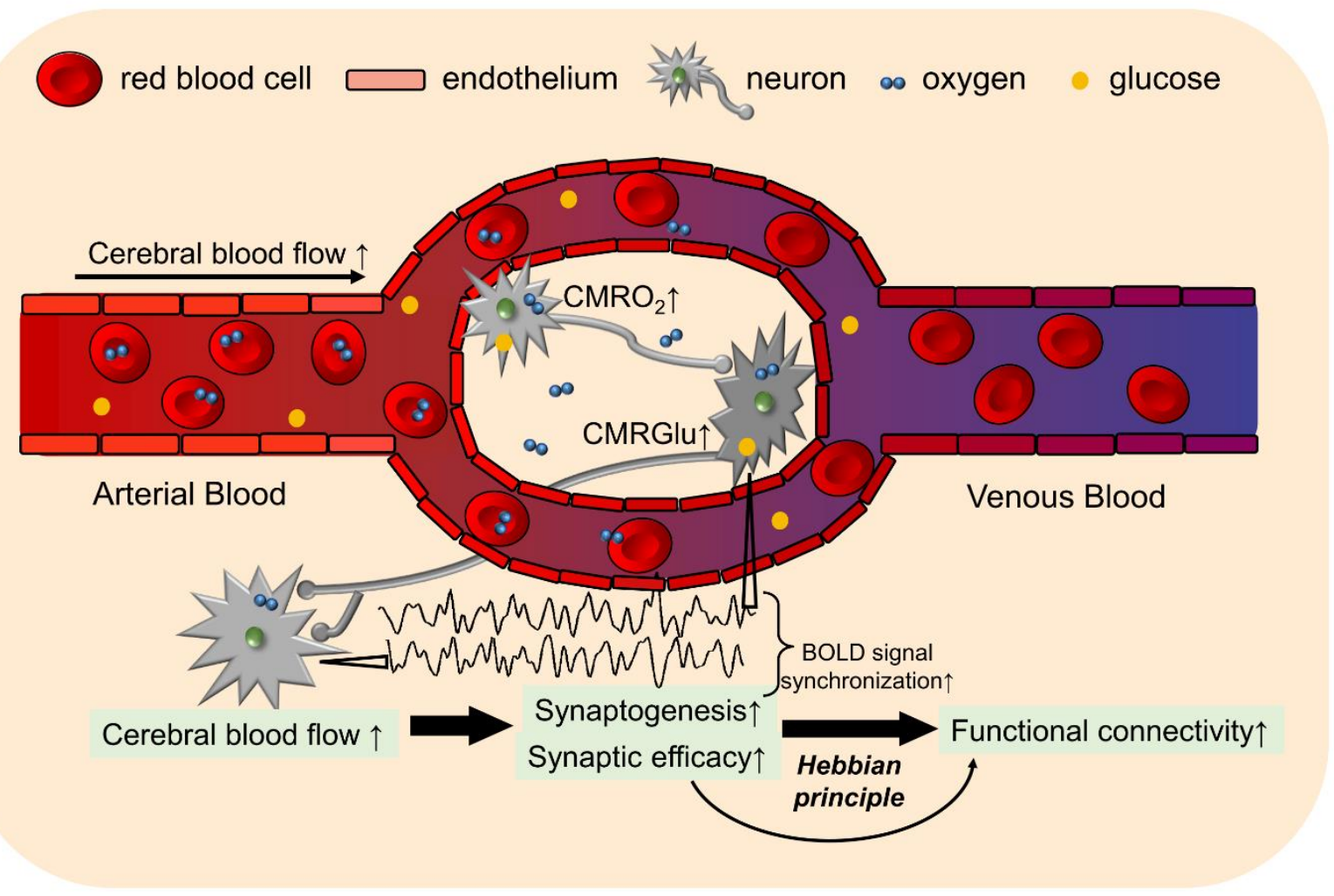

Fig. 6. A diagram illustrating hypothesized neuronal mechanism supporting coupling of regional cerebral blood flow (rCBF) and functional connectivity. Specifically, higher cerebral blood flow delivers larger amount of oxygen and glucose to neurons, leading to cascade of events of regional cerebral metabolic rate of oxygen (CMRO2) $\uparrow$ and glucose (CMRGlu) $\uparrow$, synaptogenesis $\uparrow$ and synaptic efficacy $\uparrow$, blood oxygenation level dependent (BOLD) signal synchronization $\uparrow$, and functional connectivity $\uparrow$, during infant brain development. 